\title{
Die Droge Arzt
}

\author{
«Das am allerhäufigsten verwendete Heilmittel ist der Arzt selber. Leider gibt \\ es für dieses wichtige Medikament bisher keine Pharmakologie und keine \\ Toxikologie» (M. Balint 1964; zit. nach: [1]) \\ Das soll nun endlich nachgeholt werden.
}

Bernhard Gurtner

Korrespondenz:

Dr. med. Bernhard Gurtner

Eggstrasse 76

CH-8620 Wetzikon

gurtner.bernhard@bluewin.ch

\section{ARZNEIMITTELKOMPENDIUM Supplementum $X$ \\ Medicus $^{\circledR}$}

\section{UNIVERSITAS}

\section{Zusammensetzung}

Wirkstoffe: Substantia grisea corticis cerebri, Experientia manuum, Vox humana

Hilfsstoffe: Fluidum personale, Passio professionalis, Misericordia guttata (E 707)

\section{Galenische Form und Wirkstoffmenge pro Einheit}

$\begin{array}{ll}\begin{array}{l}\text { Medicus domesticus simplex, } \\ \text { duplex oder retikularis }\end{array} & \begin{array}{l}\text { Breitband-Tropfen } \\ (11 \%)\end{array} \\ \begin{array}{l}\text { Medicus specialiter formatus } \\ \text { hochkonz. Ampullen } \\ (70 \%)\end{array} \\ \begin{array}{l}\text { Medicus clinicus dominans } \\ \text { resp. assistens }\end{array} & \text { Kapseln und Supp. } \\ \text { (nicht unbeschränkt haltbar) } & \end{array}$

\section{Indikationen/Anwendungsmöglichkeiten}

Medicus ${ }^{\circledR}$ ist indiziert bei Krankheiten, Unfällen, Suchtverhalten und gesundheitlichem Missbehagen jeglicher Art. Das Präparat wird aber auch zur Milderung von sozialen Nebenwirkungen der Marktwirtschaft und der freien Liebe empfohlen.

\section{Dosierung/Anwendung}

Die Dosierung ist abhängig von Alter, Wohnort und Versicherungsklasse der Patienten sowie vom objektiven oder subjektiven Schweregrad ihrer Probleme.

Je nach Indikation kann das Präparat unter klinischen Bedingungen mehrmals täglich, ambulant wöchentlich/monatlich in Portionen zu jeweils 5 Minuten oder alljährlich für Check-ups angewandt werden. Bei gesund Verstorbenen genügt der postterminale einmalige Einsatz eines Medicus $^{\circledR}$ simplex.

\section{Kontraindikationen}

Medicus ${ }^{\circledR}$ eignet sich schlecht für die Behandlung von Patienten, die der Schulmedizin prinzipiell misstrauen oder im Internet ein eigenes Medizinstudium abgeschlossen haben.

\section{Warnhinweise und Vorsichtmassnahmen}

Medicus ${ }^{\circledR}$ soll wenn möglich weder nachts noch am Wochenende eingesetzt werden, weil seine Wirksamkeit in diesen Perioden oft unbefriedigend ist.

Die im Medicus ${ }^{\circledR}$ enthaltene Vox humana kann bei unsorgfältigem Gebrauch schon in kleinster Menge zu akuten oder lange anhaltenden toxischen Nebenwirkungen führen. Es gibt auch allergische Reaktionen infolge Sensibilisierungen durch unangenehm empfundene frühkindliche oder postpubertäre Medicus-Applikationen.

\section{Interaktionen}

Die verschiedenen Medicus ${ }^{\circledR}$-Präparate können sich bei gleichzeitiger Anwendung potenzieren oder gegenseitig neutralisieren. Dasselbe gilt bei Beeinflussung der Patienten durch Apotheker, Komplementärmediziner oder Ratschläge in den Medien.

\section{Überdosierung}

Bekannte Phänomene sind die «surconsommation medicale» und das «Koryphäenkillersyndrom». Als Antidot gegen Medizinaltourismus eignen sich hohe Arzt- und Spitalrechnungen oder Zahlungsverweigerungen durch die Versicherungen.

\section{Eigenschaften/Wirkungen}

Doppelblindstudien lassen sich mit dieser Wirkstoffklasse nicht durchführen. Im Vergleich zu Placebos oder kontrollierter Nichtintervention hat sich das Originalpräparat meistens als signifikant überlegen erwiesen. Einige als angebliche 
Chirurgen oder Psychiater tätige Betrüger ohne jede Fachausbildung konnten sich erstaunlich lange als Generikum halten.

\section{Pharmakokinetik}

Die nach einem bewährten biologischen Verfahren erzeugten Sprösslinge werden in gymnasialen Treibhäusern zur Blüte und Reife gebracht, an Universitäten absorbiert, metabolisiert und zu unausgegorenem Saft gepresst. Nach jahrelanger Distribution und Zwischenlagerung in verschiedenen Spitälern können sie dort als Dauermedikation verbleiben oder ihre klinisch geprüfte heilsame Wirkung in der Praxis entfalten.

\section{Sonstige Hinweise} Haltbarkeit

Die Wirksamkeit des Präparates steigt mit zunehmender Anwendung und sinkt bei nachlassendem Fortbildungseifer. Die mit FMH-Kontroll- marken gekennzeichneten Originalpackungen können unbedenklich bei Zimmertemperatur gelagert werden, ihre Qualität wird bis zum Verfalldatum regelmässig überprüft und zertifiziert. Farb- und Konsistenzveränderungen im Laufe der Jahre sind normal und lassen nicht zwingend auf nachlassende Potenz schliessen. Chargen mit bitterem Geschmack sind hingegen verdächtig auf Burnout-Schäden.

\section{Hinweise für die Handhabung}

siehe Beipackbroschüre: «Wie behandle ich meinen Arzt?»

\section{Zulassungsinhaberin}

Santésuisse (demnächst in diesem Theater?)

1 Luban-Plozza B, Laederach-Hofmann K, Knaak L, Dickhaut HH. Der Arzt als Arznei. 8. Auflage, Köln: Deutscher Ärzte-Verlag; 2002. 